\title{
A multiwavelength study of a sample of Texas Radio Survey steep spectrum sources
}

\author{
Ray A. Lucas ${ }^{1}$, Neal Miller ${ }^{2}$, Anton M. Koekemoer ${ }^{1}$, \\ Jeffrey Van Duyne ${ }^{3}$ and Kenneth C. Chambers ${ }^{4}$ \\ ${ }^{1}$ Space Telescope Science Institute, 3700 San Martin Drive, Baltimore, MD 21218, USA \\ email: lucas,koekemoe@stsci.edu \\ ${ }^{2}$ National Radio Astronomy Observatory and Johns Hopkins University Department of \\ Physics and Astronomy, 3400 N. Charles Street, Baltimore, MD 21218, USA \\ email: nmiller@pha.jhu.edu \\ ${ }^{3}$ Yale University, Astronomy Department, P.O. Box 208101, New Haven, CT 06520-8101, USA \\ email: vanduyne@astro.yale.edu \\ ${ }^{4}$ Institute for Astronomy, University of Hawaii, \\ 2680 Woodlawn Road, Honolulu, HI 96822, USA \\ email: chambers@ifa.hawaii.edu
}

VLA A-array snapshots were obtained of a complete sample of steep-spectrum radio sources from the Texas Radio Interferometer survey. Though similar in sensitivity to the FIRST Survey, our A-array snapshots have better resolution, and are complementary to FIRST. All initial A-array maps are made, and we are comparing them to FIRST.

We are using multicolour Sloan Sky Survey data which covers our survey area for optical detections and identifications, in addition to DSS images. Though likely not deep enough to put useful constraints on high- $z$ objects in our sample, 2MASS survey images may be used for brighter infrared detections, and we will search for any other multiwavelength data readily available via VO resources. We aim to thus perform a multiwavelength characterization of these objects. The selection criteria have picked up many FR2-type sources (ones with bright twin lobes of radio emission.) This may be expected, since at higher fluxes, the radio source counts are dominated by powerful AGN such as FR2 galaxies, and the spectral index of their radio lobes is usually steep, thus they are bright at the low frequency used in the Texas survey observations.

There are also AGN unification models for FR2 galaxies and quasars which can be explored using this sample. Radio morphologies from our 71 VLA A-array snapshots: $\sim 43$ double-lobed, $3-4$ of the 43 show distinct core; $\sim 21$ of 43 show diffuse emission; 9 resolved; 9 point sources; 7 apparent non-detections (now being checked versus FIRST); 4 spurious (from 1989 prepublication Texas list.) Using multiwavelength data which is available for these sources, in conjunction with our radio data, we will attempt to build a database of our sample and to better understand the nature of these objects, and how they fit into various galaxy formation and evolution scenarios, and AGN unification models, etc.

\section{Acknowledgements}

We acknowledge support from the NSF, USNVO, and NRAO Jansky Fellowships, VLA staff, and 2004 NVO Summer School colleagues David Rohde and Takayuki Tamura.

\section{Reference}

Douglas, J. N. et al. 1996, AJ, 111, 1945 